\title{
THEORETICAL MODELING OF A TWO-PHASED THERMOSYPHON ASSUMING THE LIQUID RESERVATORY
}

\author{
M. A. Zanardia, \\ and N. G. C. Leiteb \\ ${ }^{\mathrm{a}}$ UNESP - FEG \\ Departamento de Energia \\ Guaratinguetá, São Paulo, Brasil \\ mzanardi@feg.unesp.br \\ $\mathrm{b}_{\text {UERJ - FAT }}$ \\ Departamento de Mecânica e Energia \\ Resende, Rio de Janeiro, Brasil \\ nleite@fat.uerj.br
}

\begin{abstract}
A theoretical modeling using the mass, momentum and energy conservation equations, about the intrinsic phenomena in the working of a cylindrical geometry two-phase thermosyphon operating on vertical was performed. The conservation equations were solved in steady-state operation for all the phases of the thermosyphon. Then model also assumed the presence of a liquid reservatory whose valves of the coefficient of heat transfer that determine the operation of functioning in the reservatory, were obtained from the correlation published in literature. The set of conservation equations was solved by using the method of finite volumes. The results achieved were checked with experimental data from literature and also from specific experiments performed in laboratory. In a general view, the theoric results matched reasonably well with those ones from the experiments, and the observed deviation were assumed by a inadequate prevision of the reservatory model used, besides keeping a stable level of the reservatory of liquid.
\end{abstract}

Keywords: two-phase thermosyphon, cylindrical thermosyphon, reservatory of liquid
NOMENCLATURE

$\mathrm{P}(\mathrm{CR})$ pressure at critical point, $\mathrm{Pa}$

Per Peclet number, dimensionless

Pr Prandtl number, dimensionless

$\mathrm{P}(\mathrm{S}) \quad$ pressure of saturation, $\mathrm{Pa}$

$\mathrm{q}$ heat transfer rate, $\mathrm{W}$

$r$ radial coordinate, $m$

$r_{\mathrm{v}} \quad$ vapor radius, $m$

Rer radial Reynolds number, dimensionless

$\mathrm{R}_{\mathrm{g}} \quad$ gas constant, $\mathrm{J} /(\mathrm{kgK})$
$\mathrm{S} \quad$ area, $\mathrm{m}^{2}$

$\mathrm{S}^{\phi} \quad$ source term

$\mathrm{t}$ time, $\mathrm{s}$

$\mathrm{T}$ temperature, $\mathrm{K}$

$\mathrm{T}(\mathrm{CR})$ temperature at critical point, $\mathrm{K}$

$\mathrm{T}(\mathrm{S})$ temperature of saturation, $\mathrm{K}$

$\mathrm{V}$ radial velocity component, $\mathrm{m} / \mathrm{s}$

V velocity vector, $\mathrm{m} / \mathrm{s}$

$\mathrm{V}(\mathrm{GCR})$ specific volume of the saturated vapor at critical point, $\mathrm{m}^{3} / \mathrm{kg}$

$\mathrm{V}_{\mathrm{r}} \quad$ reference velocity, $\mathrm{m} / \mathrm{s}$

w axial velocity component, $\mathrm{m} / \mathrm{s}$

$\mathrm{z}$ axial coordinate, $\mathrm{m}$

$\begin{array}{ll}\text { Greek } & \text { Symbols } \\ \Gamma^{\phi} & \text { diffusion coefficient } \\ \mu & \text { dynamic viscosity, } \mathrm{Ns} / \mathrm{m}^{2} \\ \rho & \text { specific mass, } \mathrm{kg} / \mathrm{m}^{3} \\ \phi & \text { variable dependent }\end{array}$

Subscripts

$\begin{array}{ll}\text { e } & \text { evaporator } \\ 1 & \text { liquid } \\ \mathrm{p} & \text { constant pressure; reservatory of liquid } \\ \mathrm{r} & \text { reference } \\ \text { sat } & \text { saturated }\end{array}$




$\begin{array}{ll}\text { v } & \text { vapor } \\ \text { w } & \text { wall } \\ \infty & \text { ambient } \\ \text { Superscripts } \\ \phi & \text { variable dependent } \\ * & \text { variable dimensionless } \\ - & \text { medium }\end{array}$

\section{INTRODUCTION}

A discussion about the mechanisms that determine the functioning of a two-phase thermosyphon will be presented, assuming it to be a vertical evacuated pipe with a reservatory of liquid on its inferior part.

The two-phase thermosyphon is better described when divided into three sections: evaporator, adiabatic section and condenser. The heat is supplied to the evaporator where there's a reservatory of liquid in the beginning of the process, this way causing a changing of phase to the vapor state. The vapor goes up by a difference in pressure and density, passing through the adiabatic section and reaching the condenser. In the condenser section, the vapor rejects the latent heat, returning to the initial liquid state, as shown in Fig. 1. The gravity force field acts onto the condensed, causing it to return to the evaporator.

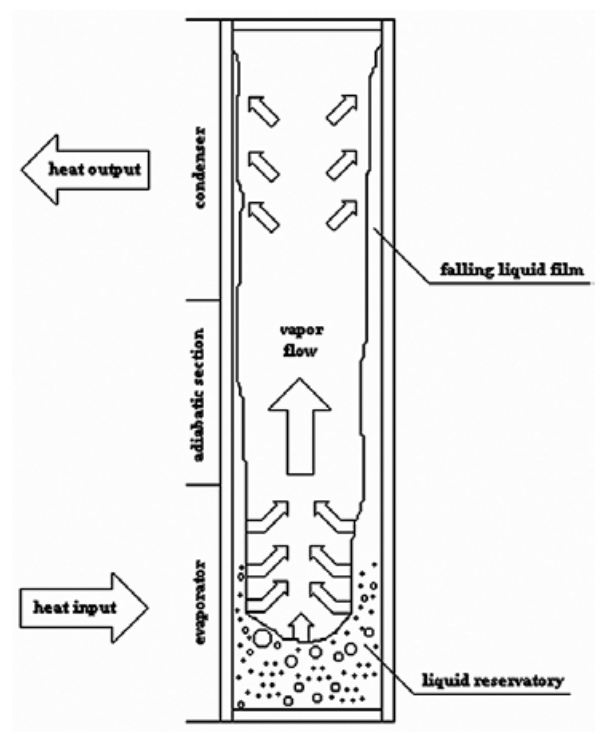

Figure 1. Conventional two-phase closed thermosyphon.

The tube thermosyphon has its ability of heat transfer due to the buoyancy force that causes the circulation, all of this joined to the pressure raise due to the changing in the saturation conditions, both caused by a temperature difference between the ends edges.
Typically, this ability is much bigger than the best conductor metal that may exist.

This device, turns to act like a super conductor heat bar. In the general, the given concept to thermosyphon takes us to a very large scale of application in practical situations in a variety of contexts such as: gas turbine blade cooling, water and soil freezing, emergency cooling in nuclear reactor, collecting and storage of solar energy, electronic components cooling, electric engine cooling and bearing machine, antiice protection, etc.

The versatility in the application associated to a reasonably easiness of a manufacturing strongly contributes to the increasing interests in theoretic modeling as well as in the perfectioning of test systems in order to perform experimental studyings. Within this context a numerical and theoretical analysis of a vertical working two phase thermosyphon, using water as working fluid will be performed. This analysis was inspired in the works of Harley and Faghri (1994), whose works didn't assume the presence of reservatory of liquid in the evaporator of the thermosyphon, this way limiting the model.

Furthermore, the complete equation of conservation that describes the conduct of the filmwise liquid are not solved, but substituted through certain simplifications based on hypothesis and transformations of coordinates, by a type of quasi-steady Nusselt solution.

This paper iniciatially proposes a complete solution of mass, momentum and energy conservation equations into the vapor nucleus, liquid film and thermosyphon wall.

During the evolution of the works, the importance of the presence of a reservatory of liquid for a better analysis of thermo-hydrodynamic phenomena occurred in the interior of the thermosyphon, turned to be more important than the transient analysis even because the experimental transient results in literature today are somewhat few if compared to the steady-state regime. Thus, it was chosen to solve the conservation equations completely but in steady-state regime, assuming the presence of reservatory of liquid in the inferior part of the pipe.

The coherence of theoric results are checked through the comparisons with experimental data obtained in literature as well as from specific tests performed in laboratory.

\section{MATHEMATICAL MODEL}

The conservation equations were applied in the complete model of a two-phase thermosyphon on vertical operation, a cylindrical geometric shape and using water as fluid of work. These equations were used as described in Ganic, Hartnett and Rohsenow (1985) form, in bidimensional and cylindrical coordinates, with its respective hypothesis, and were effectively solved, and their results compared to exper- 
imental data obtained from the UNESP - Research Laboratory of the Engineering College from Guaratinguetá Campus - SP - Brazil, as well as through specialized literature.

For the vapor phase is given:

Mass conservation:

$$
\frac{\partial \rho}{\partial t}+\nabla \cdot(\rho \mathbf{V})=0
$$

Momentum conservation:

$$
\rho \frac{\mathrm{DV}}{\mathrm{Dt}}=-\nabla \mathrm{p}+\frac{1}{3} \mu \nabla(\nabla . \mathbf{V})+\mu \nabla^{2} \mathbf{V}+\rho \mathrm{g}
$$

Energy conservation:

$\rho c_{\mathrm{p}} \frac{\mathrm{DT}}{\mathrm{Dt}}-\nabla . \mathrm{k} \nabla \mathrm{T}-\frac{\mathrm{Dp}}{\mathrm{Dt}}-\mu \phi=0$

with

$$
\begin{aligned}
\phi=2\left[\left(\frac{\partial \mathrm{v}}{\partial \mathrm{r}}\right)^{2}+\left(\frac{\mathrm{v}}{\mathrm{r}}\right)^{2}+\left(\frac{\partial \mathrm{w}}{\partial \mathrm{z}}\right)^{2}\right]+\left(\frac{\partial \mathrm{v}}{\partial \mathrm{z}}+\right. & \left.\frac{\partial \mathrm{w}}{\partial \mathrm{r}}\right)^{2}+ \\
& -\frac{2}{3}\left[\frac{1}{\mathrm{r}} \frac{\partial(\mathrm{rv})}{\partial \mathrm{r}}+\frac{\partial \mathrm{w}}{\partial \mathrm{z}}\right]^{2}
\end{aligned}
$$

The state equation is given by:

$$
\mathrm{p}=\rho \mathrm{R}_{\mathrm{g}} \mathrm{T}
$$

At the pipe wall, the bidimensional and transient energy conservation equation is written as:

$$
\rho c_{\mathrm{p}} \frac{\partial \mathrm{T}}{\partial \mathrm{t}}=\mathrm{k}\left[\frac{1}{\mathrm{r}} \frac{\partial}{\partial \mathrm{r}}\left(\mathrm{r} \frac{\partial \mathrm{T}}{\partial \mathrm{r}}\right)+\frac{\partial^{2} \mathrm{~T}}{\partial \mathrm{z}^{2}}\right]
$$

To analyse the liquid film, assuming transient flow, laminar, incompressible and constants properties, the bidimensional conservation equations in cylindrical coordinates can be described as:

Mass conservation:

$$
\frac{1}{\mathrm{r}} \frac{\partial}{\partial \mathrm{r}}(\mathrm{rv})+\frac{\partial \mathrm{w}}{\partial \mathrm{z}}=0
$$

Momentum conservation:

Axial direction:

$$
\begin{aligned}
\rho\left(\frac{\partial \mathrm{w}}{\partial \mathrm{t}}+\mathrm{v} \frac{\partial \mathrm{w}}{\partial \mathrm{r}}+\mathrm{w} \frac{\partial \mathrm{w}}{\partial \mathrm{z}}\right)=-\frac{\partial \mathrm{p}}{\partial \mathrm{z}}+ \\
+\mu\left[\frac{1}{\mathrm{r}} \frac{\partial}{\partial \mathrm{r}}\left(\mathrm{r} \frac{\partial \mathrm{w}}{\partial \mathrm{r}}\right)+\frac{\partial^{2} \mathrm{w}}{\partial \mathrm{z}^{2}}\right]+\rho \mathrm{g}
\end{aligned}
$$

Radial direction:

$$
\begin{aligned}
& \rho\left(\frac{\partial \mathrm{v}}{\partial \mathrm{t}}+\mathrm{v} \frac{\partial \mathrm{v}}{\partial \mathrm{r}}+\mathrm{w}\right.\left.\frac{\partial \mathrm{v}}{\partial \mathrm{z}}\right)=-\frac{\partial \mathrm{p}}{\partial \mathrm{r}}+ \\
&+\mu\left[\frac{1}{\mathrm{r}} \frac{\partial}{\partial \mathrm{r}}\left(\mathrm{r} \frac{\partial \mathrm{v}}{\partial \mathrm{r}}\right)-\frac{\mathrm{v}}{\mathrm{r}^{2}}+\frac{\partial^{2} \mathrm{v}}{\partial \mathrm{z}^{2}}\right]
\end{aligned}
$$

Energy conservation:

$$
\rho c_{\mathrm{p}}\left(\frac{\partial \mathrm{T}}{\partial \mathrm{t}}+\mathrm{v} \frac{\partial \mathrm{T}}{\partial \mathrm{r}}+\mathrm{w} \frac{\partial \mathrm{T}}{\partial \mathrm{z}}\right)=\frac{1}{\mathrm{r}} \frac{\partial}{\partial \mathrm{r}}\left(\mathrm{rk} \frac{\partial \mathrm{T}}{\partial \mathrm{r}}\right)+\frac{\partial}{\partial \mathrm{z}}\left(\mathrm{k} \frac{\partial \mathrm{T}}{\partial \mathrm{z}}\right)
$$

The conservation equations were rightly adimensionalized through the following :

$$
\begin{aligned}
& \mathrm{z}^{*}=\frac{\mathrm{z}}{\mathrm{L}} \quad \mathrm{w}^{*}=\frac{\mathrm{w}}{\mathrm{V}_{\mathrm{r}}} \rho^{*}=\frac{\rho}{\rho_{\mathrm{r}}} \quad \mathrm{p}^{*}=\frac{\mathrm{p}}{\rho_{\mathrm{r}} \mathrm{V}_{\mathrm{r}}^{2}} \\
& \mathrm{r}^{*}=\frac{\mathrm{r}}{\mathrm{r}_{\mathrm{v}}} \quad \mathrm{v}^{*}=\frac{\mathrm{v}}{\mathrm{V}_{\mathrm{r}}} \mu^{*}=\frac{\mu}{\mu_{\mathrm{r}}} \quad \mathrm{t}^{*}=\frac{\mathrm{t}}{\mathrm{r}_{\mathrm{v}} / \mathrm{V}_{\mathrm{r}}}
\end{aligned}
$$

At the varying temperature, it wasn't performed any adimensionalization for this could cause an extra difficulty in the solution process due to the adopted boundary conditions. During the adimensionalization, some adimensional groups occurred:

$\operatorname{Rer}=\frac{\rho_{\mathrm{r}} \mathrm{V}_{\mathrm{r}} \mathrm{r}_{\mathrm{v}}}{\mu_{\mathrm{r}}} \quad \operatorname{Pr}=\frac{\mu_{\mathrm{r}} \mathrm{c}_{\mathrm{p}}}{\mathrm{k}} \quad$ Per $=$ Rer.Pr

We have, then, the following equations according to the phases:

Mass conservation (vapor phase):

$\frac{1}{\mathrm{r}^{*}}\left[\mathrm{r}^{*} \frac{\partial \rho^{*}}{\partial \mathrm{t}^{*}}+\frac{\partial}{\partial \mathrm{r}^{*}}\left(\mathrm{r}^{*} \rho^{*} \mathrm{v}^{*}\right)+\frac{\mathrm{r}_{\mathrm{v}}}{\mathrm{L}} \frac{\partial}{\partial \mathrm{z}^{*}}\left(\mathrm{r}^{*} \rho^{*} \mathrm{w}^{*}\right)\right]=0$

Momentum conservation (vapor phase):

Axial direction:

$$
\begin{aligned}
& \frac{1}{\mathrm{r}^{*}}\left[\mathrm{r}^{*} \frac{\partial}{\partial \mathrm{t}^{*}}\left(\rho^{*} \mathrm{w}^{*}\right)+\frac{\partial}{\partial \mathrm{r}^{*}}\left(\mathrm{r}^{*} \rho^{*} \mathrm{v}^{*} \mathrm{w}^{*}\right)+\frac{\mathrm{r}_{\mathrm{v}}}{\mathrm{L}} \frac{\partial}{\partial \mathrm{z}^{*}}\left(\mathrm{r}^{*} \rho^{*} \mathrm{w}^{*} \mathrm{w}^{*}\right)\right]= \\
& \frac{1}{\mathrm{r}^{*}}\left[\frac{\partial}{\partial \mathrm{r}^{*}}\left(\frac{\mu^{*} \mathrm{r}^{*}}{\operatorname{Re} \mathrm{r}} \frac{\partial \mathrm{w}^{*}}{\partial \mathrm{r}^{*}}\right)+\left(\frac{\mathrm{r}_{\mathrm{v}}}{\mathrm{L}}\right)^{2} \frac{\partial}{\partial \mathrm{z}^{*}}\left(\frac{\mu^{*} \mathrm{r}^{*}}{\operatorname{Rer}} \frac{\partial \mathrm{w}^{*}}{\partial \mathrm{z}^{*}}\right)\right]+ \\
& +\frac{\mathrm{w}^{*}}{\mathrm{r}^{*}}\left[\mathrm{r}^{*} \frac{\partial \rho^{*}}{\partial \mathrm{t}^{*}}+\frac{\partial}{\partial \mathrm{r}^{*}}\left(\mathrm{r}^{*} \rho^{*} \mathrm{v}^{*}\right)+\frac{\mathrm{r}_{\mathrm{v}}}{\mathrm{L}} \frac{\partial}{\partial \mathrm{z}^{*}}\left(\mathrm{r}^{*} \rho^{*} \mathrm{w}^{*}\right)\right]-\frac{\mathrm{r}_{\mathrm{v}}}{\mathrm{L}} \frac{\partial \mathrm{p}^{*}}{\partial \mathrm{z}^{*}}+
\end{aligned}
$$




$$
\begin{aligned}
& +\frac{1}{3} \frac{1}{\operatorname{Rer}}\left\{\frac{\mathrm{r}_{\mathrm{v}}}{\mathrm{L}} \frac{\partial}{\partial \mathrm{z}^{*}}\left[\frac{\mu^{*}}{\mathrm{r}^{*}} \frac{\partial}{\partial \mathrm{r}^{*}}\left(\mathrm{r}^{*} \mathrm{v}^{*}\right)\right]+\left(\frac{\mathrm{r}_{\mathrm{v}}}{\mathrm{L}}\right)^{2} \frac{\partial}{\partial \mathrm{z}^{*}}\left(\mu^{*} \frac{\partial \mathrm{w}^{*}}{\partial \mathrm{z}^{*}}\right)\right\}+ \\
& +\left(\frac{\mathrm{r}_{\mathrm{v}} \mathrm{g}_{\mathrm{z}}}{\mathrm{V}_{\mathrm{r}}^{2}}\right) \rho^{*}
\end{aligned}
$$

Radial direction:

$$
\begin{gathered}
\frac{1}{\mathrm{r}^{*}}\left[\mathrm{r}^{*} \frac{\partial}{\partial \mathrm{t}^{*}}\left(\rho^{*} \mathrm{v}^{*}\right)+\frac{\partial}{\partial \mathrm{r}^{*}}\left(\mathrm{r}^{*} \rho^{*} \mathrm{v}^{*} \mathrm{v}^{*}\right)+\frac{\mathrm{r}_{\mathrm{v}}}{\mathrm{L}} \frac{\partial}{\partial \mathrm{z}^{*}}\left(\mathrm{r}^{*} \rho^{*} \mathrm{w}^{*} \mathrm{v}^{*}\right)\right]= \\
\frac{1}{\mathrm{r}^{*}}\left[\frac{\partial}{\partial \mathrm{r}^{*}}\left(\frac{\mu^{*} \mathrm{r}^{*}}{\operatorname{Re} \mathrm{r}} \frac{\partial \mathrm{v}^{*}}{\partial \mathrm{r}^{*}}\right)+\left(\frac{\mathrm{r}_{\mathrm{v}}}{\mathrm{L}}\right)^{2} \frac{\partial}{\partial \mathrm{z}^{*}}\left(\frac{\mu^{*} \mathrm{r}^{*}}{\operatorname{Rer} \mathrm{r} \mathrm{v}^{*}}\right)\right]+ \\
+\frac{\mathrm{v}^{*}}{\mathrm{r}^{*}}\left[\mathrm{r}^{*} \frac{\partial \rho^{*}}{\partial \mathrm{t}^{*}}+\frac{\partial}{\partial \mathrm{r}^{*}}\left(\mathrm{r}^{*} \rho^{*} \mathrm{v}^{*}\right)+\frac{\mathrm{r}_{\mathrm{v}}}{\mathrm{L}} \frac{\partial}{\partial \mathrm{z}^{*}}\left(\mathrm{r}^{*} \rho^{*} \mathrm{w}^{*}\right)\right]+ \\
-\frac{\mathrm{v}^{*}}{\mathrm{r}^{*}}\left[\frac{\mu^{*}}{\operatorname{Rer} \mathrm{r}^{*}}\right]-\frac{\partial \mathrm{p}^{*}}{\partial \mathrm{r}^{*}}+\frac{1}{3} \frac{1}{\operatorname{Rer}}\left\{\frac{\partial}{\partial \mathrm{r}^{*}}\left[\frac{\mu^{*}}{\mathrm{r}^{*}} \frac{\partial}{\partial \mathrm{r}^{*}}\left(\mathrm{r}^{*} \mathrm{v}^{*}\right)\right]\right\}+ \\
+\frac{1}{3} \frac{1}{\operatorname{Re} \mathrm{r}}\left\{\left(\frac{\mathrm{r} \mathrm{v}}{\mathrm{L}}\right) \frac{\partial}{\partial \mathrm{r}^{*}}\left(\mu^{*} \frac{\partial \mathrm{w}^{*}}{\partial \mathrm{z}^{*}}\right)\right\}
\end{gathered}
$$

Energy conservation (vapor phase):

$$
\begin{aligned}
& \frac{1}{r^{*}}\left[r^{*} \frac{\partial}{\partial t^{*}}\left(\rho^{*} T\right)+\frac{\partial}{\partial r^{*}}\left(r^{*} \rho^{*} v^{*} T\right)+\frac{r_{v}}{L} \frac{\partial}{\partial z^{*}}\left(r^{*} \rho^{*} w^{*} T\right)\right]= \\
& \frac{1}{\mathrm{r}^{*}}\left[\frac{\partial}{\partial \mathrm{r}^{*}}\left(\frac{\mathrm{r}^{*}}{\operatorname{Per}} \frac{\partial \mathrm{T}}{\partial \mathrm{r}^{*}}\right)+\left(\frac{\mathrm{r}_{\mathrm{v}}}{\mathrm{L}}\right)^{2} \frac{\partial}{\partial \mathrm{z}^{*}}\left(\frac{\mathrm{r}^{*}}{\operatorname{Per}} \frac{\partial \mathrm{T}}{\partial \mathrm{z}^{*}}\right)\right]+ \\
& +\frac{\mathrm{T}}{\mathrm{r}^{*}}\left[\mathrm{r}^{*} \frac{\partial \rho^{*}}{\partial \mathrm{t}^{*}}+\frac{\partial}{\partial \mathrm{r}^{*}}\left(\mathrm{r}^{*} \rho^{*} \mathrm{v}^{*}\right)+\frac{\mathrm{r}_{\mathrm{v}}}{\mathrm{L}} \frac{\partial}{\partial \mathrm{z}^{*}}\left(\mathrm{r}^{*} \rho^{*} \mathrm{w}^{*}\right)\right]+ \\
& +\frac{\mathrm{V}_{\mathrm{r}}^{2}}{\mathrm{c}_{\mathrm{p}}}\left[\frac{\partial \mathrm{p}^{*}}{\partial \mathrm{t}^{*}}+\mathrm{v}^{*} \frac{\partial \mathrm{p}^{*}}{\partial \mathrm{r}^{*}}+\frac{\mathrm{r}_{\mathrm{v}}}{\mathrm{L}} \mathrm{w}^{*} \frac{\partial \mathrm{p}^{*}}{\partial \mathrm{z}^{*}}\right]+ \\
& +\frac{\mu^{*} \mathrm{~V}_{\mathrm{r}}^{2}}{\operatorname{Rer} \mathrm{c}_{\mathrm{p}}}\left\{2\left[\left(\frac{\partial \mathrm{v}^{*}}{\partial \mathrm{r}^{*}}\right)^{2}+\left(\frac{\mathrm{v}^{*}}{\mathrm{r}^{*}}\right)^{2}+\left(\frac{\mathrm{r}_{\mathrm{v}}}{\mathrm{L}} \frac{\partial \mathrm{w}^{*}}{\partial \mathrm{z}^{*}}\right)^{2}\right]\right\}+ \\
& +\frac{\mu^{*} V_{r}^{2}}{\operatorname{Rer} c_{p}}\left[\left(\frac{r_{v}}{L} \frac{\partial v^{*}}{\partial z^{*}}\right)^{2}+2 \frac{r_{v}}{L} \frac{\partial v^{*}}{\partial z^{*}} \frac{\partial w^{*}}{\partial r^{*}}+\left(\frac{\partial w^{*}}{\partial r^{*}}\right)^{2}\right]+ \\
& \frac{\mu^{*} V_{r}^{2}}{\operatorname{Rerc} c_{p}}\left\{\frac{2}{3}\left[\left(\frac{1}{r^{*}} \frac{\partial}{\partial r^{*}}\left(r^{*} v^{*}\right)\right)^{2}\right]\right\}+ \\
& -\frac{\mu^{*} V_{r}^{2}}{\operatorname{Rer} c_{p}}\left\{\frac{2}{3}\left[2 \frac{r_{v}}{L} \frac{1}{r^{*}} \frac{\partial}{\partial r^{*}}\left(r^{*} v^{*}\right) \frac{\partial w^{*}}{\partial z^{*}}\right]\right\}+ \\
& -\frac{\mathrm{V}_{\mathrm{r}}^{2}}{\operatorname{Rer} \mathrm{c}_{\mathrm{p}}}\left\{\frac{2}{3}\left[\left(\frac{\mathrm{r}_{\mathrm{v}}}{\mathrm{L}} \frac{\partial \mathrm{w}^{*}}{\partial \mathrm{z}^{*}}\right)^{2}\right]\right\}
\end{aligned}
$$

This equations systems are complete using the state equation for an ideal gas in the adimensional form:

$$
\mathrm{p}^{*}=\left(\frac{1}{\mathrm{~V}_{\mathrm{r}}^{2}}\right) \rho^{*} \mathrm{R}_{\mathrm{g}} \mathrm{T}
$$

This equation has the density value during the process of numerical solution. An another way presented by Irvine and Liley (1984), of calculating the density value was also used and compared to the Eq.(15), concordance showing a very good acceptation.

The way Irvine and Liley (1984) calculate density, was adopted because it follows the same reasoning of the expression used in calculating the temperature of saturation because of the pressure, which also became necessary during the process of solution. Thus, the expressions adopted for the implantation of the density calculus decurrent of temperature and pressure and temperature of saturation decurrent of pressure, were the following:

$$
\begin{aligned}
& \rho_{\mathrm{v}}=\frac{\mathrm{P}(\mathrm{S})}{\mathrm{P}(\mathrm{CR}) \cdot \mathrm{V}(\mathrm{GCR}) \cdot \mathrm{Y}(\mathrm{S})} \\
& \mathrm{T}_{\mathrm{sat}}=\mathrm{A}+\frac{\mathrm{B}}{\ln [\mathrm{P}(\mathrm{S})]+\mathrm{C}}
\end{aligned}
$$

with

$$
\begin{gathered}
Y(S)=A+B \cdot T(C)^{\frac{1}{3}}+C \cdot T(C)^{\frac{5}{6}}+D \cdot T(C)^{\frac{7}{8}}+\sum_{n=1}^{7} E(n) \cdot T(C)^{n} \\
T(C)=\frac{T(C R)-T(S)}{T(C R)}
\end{gathered}
$$

As to the liquid film:

Mass conservation (liquid phase):

$$
\frac{1}{r^{*}}\left[\frac{\partial}{\partial r^{*}}\left(r^{*} \rho^{*} v^{*}\right)+\frac{r_{v}}{L} \frac{\partial}{\partial z^{*}}\left(r^{*} \rho^{*} w^{*}\right)\right]=0
$$

Momentum conservation (liquid phase): Axial direction:

$$
\begin{gathered}
\frac{1}{r^{*}}\left[r^{*} \frac{\partial}{\partial t^{*}}\left(\rho^{*} w^{*}\right)+\frac{\partial}{\partial r^{*}}\left(r^{*} \rho^{*} v^{*} w^{*}\right)+\frac{r_{v}}{L} \frac{\partial}{\partial z^{*}}\left(r^{*} \rho^{*} w^{*} w^{*}\right)\right]= \\
\frac{1}{r^{*}}\left[\frac{\partial}{\partial r^{*}}\left(\frac{\mu^{*} r^{*}}{\operatorname{Rer}} \frac{\partial w^{*}}{\partial r^{*}}\right)+\left(\frac{r_{v}}{L}\right)^{2} \frac{\partial}{\partial z^{*}}\left(\frac{\mu^{*} r^{*}}{\operatorname{Rer}} \frac{\partial w^{*}}{\partial z^{*}}\right)\right]+
\end{gathered}
$$




$$
\begin{aligned}
& +\frac{\mathrm{w}^{*}}{\mathrm{r}^{*}}\left[\frac{\partial}{\partial \mathrm{r}^{*}}\left(\mathrm{r}^{*} \rho^{*} \mathrm{v}^{*}\right)+\frac{\mathrm{r}_{\mathrm{v}}}{\mathrm{L}} \frac{\partial}{\partial \mathrm{z}^{*}}\left(\mathrm{r}^{*} \rho^{*} \mathrm{w}^{*}\right)\right]-\frac{\mathrm{r}_{\mathrm{v}}}{\mathrm{L}} \frac{\partial \mathrm{p}^{*}}{\partial \mathrm{z}^{*}}+ \\
& +\left(\frac{\mathrm{r}_{\mathrm{v}} \mathrm{g}_{\mathrm{z}}}{\mathrm{V}_{\mathrm{r}}^{2}}\right) \rho^{*}
\end{aligned}
$$

Radial direction:

$$
\begin{gathered}
\frac{1}{\mathrm{r}^{*}}\left[\mathrm{r}^{*} \frac{\partial}{\partial \mathrm{t}^{*}}\left(\rho^{*} \mathrm{v}^{*}\right)+\frac{\partial}{\partial \mathrm{r}^{*}}\left(\mathrm{r}^{*} \rho^{*} \mathrm{v}^{*} \mathrm{v}^{*}\right)+\frac{\mathrm{r}_{\mathrm{v}}}{\mathrm{L}} \frac{\partial}{\partial \mathrm{z}^{*}}\left(\mathrm{r}^{*} \rho^{*} \mathrm{w}^{*} \mathrm{v}^{*}\right)\right]= \\
\frac{1}{\mathrm{r}^{*}}\left[\frac{\partial}{\partial \mathrm{r}^{*}}\left(\frac{\mu^{*} \mathrm{r}^{*}}{\operatorname{Rer}} \frac{\partial \mathrm{v}^{*}}{\partial \mathrm{r}^{*}}\right)+\left(\frac{\mathrm{r}_{\mathrm{v}}}{\mathrm{L}}\right)^{2} \frac{\partial}{\partial \mathrm{z}^{*}}\left(\frac{\mu^{*} \mathrm{r}^{*}}{\operatorname{Rer}} \frac{\partial \mathrm{v}^{*}}{\partial \mathrm{z}^{*}}\right)\right]+ \\
+\frac{\mathrm{v}^{*}}{\mathrm{r}^{*}}\left[\frac{\partial}{\partial \mathrm{r}^{*}}\left(\mathrm{r}^{*} \rho^{*} \mathrm{v}^{*}\right)+\frac{\mathrm{r}_{\mathrm{v}}}{\mathrm{L}} \frac{\partial}{\partial \mathrm{z}^{*}}\left(\mathrm{r}^{*} \rho^{*} \mathrm{w}^{*}\right)-\frac{\mu^{*}}{\operatorname{Rer} \mathrm{r}^{*}}\right]-\frac{\partial \mathrm{p}^{*}}{\partial \mathrm{r}^{*}}
\end{gathered}
$$

Energy conservation (liquid phase):

$$
\begin{gathered}
\frac{1}{\mathrm{r}^{*}}\left[\mathrm{r}^{*} \frac{\partial}{\partial \mathrm{t}^{*}}\left(\rho^{*} \mathrm{~T}\right)+\frac{\partial}{\partial \mathrm{r}^{*}}\left(\mathrm{r}^{*} \rho^{*} \mathrm{v}^{*} \mathrm{~T}\right)+\frac{\mathrm{r}_{\mathrm{v}}}{\mathrm{L}} \frac{\partial}{\partial \mathrm{z}^{*}}\left(\mathrm{r}^{*} \rho^{*} \mathrm{w}^{*} \mathrm{~T}\right)\right]= \\
\frac{1}{\mathrm{r}^{*}}\left[\frac{\partial}{\partial \mathrm{r}^{*}}\left(\frac{\mathrm{r}^{*}}{\operatorname{Per}} \frac{\partial \mathrm{T}}{\partial \mathrm{r}^{*}}\right)+\left(\frac{\mathrm{r}_{\mathrm{v}}}{\mathrm{L}}\right)^{2} \frac{\partial}{\partial \mathrm{z}^{*}}\left(\frac{\mathrm{r}^{*}}{\operatorname{Per}} \frac{\partial \mathrm{T}}{\partial \mathrm{z}^{*}}\right)\right]+ \\
+\frac{\mathrm{T}}{\mathrm{r}^{*}}\left[\frac{\partial}{\partial \mathrm{r}^{*}}\left(\mathrm{r}^{*} \rho^{*} \mathrm{v}^{*}\right)+\frac{\mathrm{r}_{\mathrm{v}}}{\mathrm{L}} \frac{\partial}{\partial \mathrm{z}^{*}}\left(\mathrm{r}^{*} \rho^{*} \mathrm{w}^{*}\right)\right]
\end{gathered}
$$

At pipe wall there is:

Energy conservation (solid phase):

$$
\begin{gathered}
\frac{1}{\mathrm{r}^{*}}\left[\mathrm{r}^{*} \frac{\partial}{\partial \mathrm{t}^{*}}\left(\rho^{*} \mathrm{~T}\right)\right]= \\
\frac{1}{\mathrm{r}^{*}}\left[\frac{\partial}{\partial \mathrm{r}^{*}}\left(\frac{\mathrm{r}^{*}}{\operatorname{Per}} \frac{\partial \mathrm{T}}{\partial \mathrm{r}^{*}}\right)+\left(\frac{\mathrm{r}_{\mathrm{v}}}{\mathrm{L}}\right)^{2} \frac{\partial}{\partial \mathrm{z}^{*}}\left(\frac{\mathrm{r}^{*}}{\operatorname{Per}} \frac{\partial \mathrm{T}}{\partial \mathrm{z}^{*}}\right)\right]
\end{gathered}
$$

It's observed that the mass conservation equation was maintained in the conservation equation of the momentum and energy in the liquid and vapor phase, and during the interactive process of solution before of convergence, the continuity equation will not be necessarily satisfied. However, when it's found the solution for the velocity field, these terms are annulled.
The conservation equation presented in the adimensional form can be described to a general scalar field of a variable dependent $\phi$, as :

$$
\begin{gathered}
\frac{1}{\mathrm{r}^{*}}\left[\mathrm{r}^{*} \frac{\partial}{\partial \mathrm{t}^{*}}\left(\rho^{*} \phi\right)+\frac{\partial}{\partial \mathrm{r}^{*}}\left(\mathrm{r}^{*} \rho^{*} \mathrm{v}^{*} \phi\right)+\frac{\mathrm{r}_{\mathrm{v}}}{\mathrm{L}} \frac{\partial}{\partial \mathrm{z}^{*}}\left(\mathrm{r}^{*} \rho^{*} \mathrm{w}^{*} \phi\right)\right]= \\
\frac{1}{\mathrm{r}^{*}}\left[\frac{\partial}{\partial \mathrm{r}^{*}}\left(\Gamma^{\phi} \mathrm{r}^{*} \frac{\partial \phi}{\partial \mathrm{r}^{*}}\right)+\left(\frac{\mathrm{r}_{\mathrm{v}}}{\mathrm{L}}\right)^{2} \frac{\partial}{\partial \mathrm{z}^{*}}\left(\Gamma^{\phi} \mathrm{r}^{*} \frac{\partial \phi}{\partial \mathrm{z}^{*}}\right)\right]+\mathrm{S}^{\phi}
\end{gathered}
$$

The Eq.(23) form where the fluxes occur inside the signal of the derivative, named conservative form or divergent, makes easy the obtaining of approximated equations for the method of finite volumes, besides allowing the use of the same process of solutions for all the variables. There are two ways of obtaining the approximated equations in the finite volume method. Through the performance of balancing of the properties in the elementary volumes (or finite volumes) or going from the differential equation in the conservative form and then integrating it on the elementar volume, in space and time. The way of presentation chosen on this paper was the one of obtaining the approximated equations integrating the differential equation because this is the simplest way.

For the application of the method of the control volumes developed by Patankar (1980), there's the necessity to promote the discretization of the property of interest, dividing it into elementary cells. Then, a structured discretization - built by using a coordinate system - through the cylindrical coordinate was done, obtaining this way the property of discretized calculus for investigation.

The application of numerical method in this focus, requires now specifics boundary conditions to the dominant equations, so this way can be solved the system of equations.

This way, to enable the comprehension of application of boundary conditions, Fig. 2 presents the dominion of calculus with its respective boundary conditions, divided by areas.

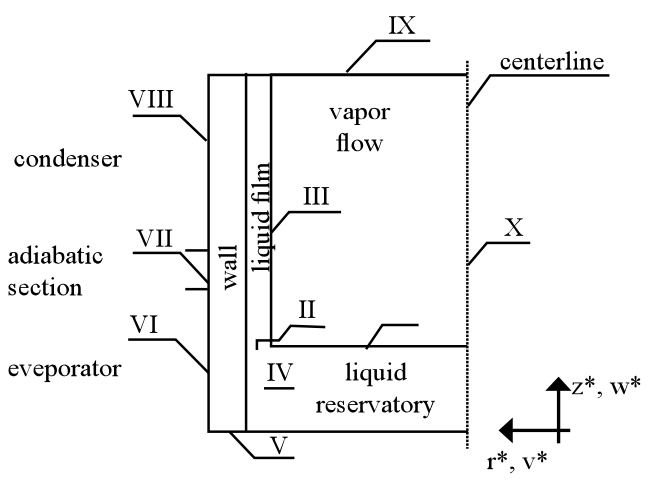

Figure 2. Dominion of axisymetric calculus. 


\section{BOUNDARY CONDITIONS}

Region I: interfacial reservatory of liquid nucleus of vapor

$$
\begin{gathered}
\mathrm{W}^{*}=\frac{\mathrm{Q}_{\mathrm{p}}}{\mathrm{V}_{\mathrm{r}} \rho_{\mathrm{v}} \mathrm{S}_{\mathrm{p}} \mathrm{h}_{\mathrm{fg}}} \\
\mathrm{T}=\mathrm{T}_{\mathrm{sat}}(\mathrm{p})
\end{gathered}
$$

The velocity was calculated assuming that all the energy in the reservatory of liquid, was used in its evaporation, i.e., only the latent heat was considered. The temperature was calculated in each point of interfacial, based on correspondent pressure.

\section{Region II: interface reservatory of liquid film of liquid}

$$
\begin{aligned}
\mathrm{w}^{*} & =\frac{\rho_{\mathrm{v}} \mathrm{w}_{\mathrm{v}} \mathrm{S}_{\mathrm{v}}}{\mathrm{V}_{\mathrm{r}} \rho_{\mathrm{l}} \mathrm{S}_{1}} \\
\mathrm{~T} & =\overline{\mathrm{T}}_{\text {sat }}(\mathrm{p})
\end{aligned}
$$

At this interfacial the velocity was calculated from the balancing of mass in the transversal section, while the temperature was based on a medium pressure of the vapor nucleus region.

Region III: liquid vapor interface film

$$
\begin{gathered}
\mathrm{v}^{*}=\frac{-\mathrm{k}_{1} \frac{\partial \mathrm{T}_{1}}{\partial \mathrm{r}^{*}}+\mathrm{k}_{\mathrm{v}} \frac{\partial \mathrm{T}_{\mathrm{v}}}{\partial \mathrm{r}^{*}}}{\mathrm{~V}_{\mathrm{r}} \rho_{\mathrm{r}} \mathrm{r}_{\mathrm{v}} \rho^{*} \mathrm{~h}_{\mathrm{fg}}} \\
\mathrm{T}=\mathrm{T}_{\mathrm{sat}}(\mathrm{p})
\end{gathered}
$$

A balancing of energy assuming conduction and latent heat was performed on interfacial nucleus of vapor-film of liquid, to obtain the radial velocity. The temperatures were calculated as in Region I.

\section{Region IV: interfacial reservatory of liquid and film of} the pipe-wall liquid

$$
\begin{gathered}
\mathrm{w}^{*}=0 \\
\mathrm{v}^{*}=0 \\
\mathrm{~T}=\frac{\mathrm{q}_{\mathrm{e}}}{\mathrm{h}_{\mathrm{p}}}+\mathrm{T}_{\mathrm{p}}
\end{gathered}
$$

The condition of no-slip was adopted to the components of velocity in all the interface, while for the temperature in the reservatory region, it was considered a known temperature calculated from Newton's cooling law. For the rest of interface, a continuity of temperature was admitted as boundary condition.

\section{Region V: liquid wall interface-environment}

$$
\frac{\partial \mathrm{T}}{\partial \mathrm{z}^{*}}=0
$$

Region VI: evaporator

$$
\frac{\partial \mathrm{T}}{\partial \mathrm{r}^{*}}=\frac{\mathrm{r}_{\mathrm{v}} \mathrm{q}_{\mathrm{e}}}{\mathrm{k}_{\mathrm{w}}}
$$

\section{Region VII: adiabatic section}

$$
\frac{\partial \mathrm{T}}{\partial \mathrm{r}^{*}}=0
$$

Region VIII: condensator

$$
\frac{\partial \mathrm{T}}{\partial \mathrm{r}^{*}}=-\frac{\mathrm{r}_{\mathrm{v}} \mathrm{h}_{\infty}}{\mathrm{k}_{\mathrm{w}}}\left(\mathrm{T}_{\mathrm{o}}-\mathrm{T}_{\infty}\right)
$$

\section{Region IX: condenser end cap}

$$
\begin{aligned}
& \mathrm{w}^{*}=0 \\
& \mathrm{v}^{*}=0 \\
& \frac{\partial \mathrm{T}}{\partial \mathrm{z}^{*}}=0
\end{aligned}
$$

\section{Region X: centerline}

$$
\begin{gathered}
\frac{\partial \mathrm{w}^{*}}{\partial \mathrm{r}^{*}}=0 \\
\mathrm{v}^{*}=0 \\
\frac{\partial \mathrm{T}}{\partial \mathrm{r}^{*}}=0
\end{gathered}
$$

The value of temperature of reservatory $T_{p}$, was calculated through the adding of the medium temperature of saturation on the interface of reservatory of nucleus-liquid vapor plus the raising of temperature caused by hydrostatic pressure of the reservatory of liquid. The coefficient value of heat transfer by convection $h_{p}$, had its value obtained through the expressions developed by El-Genk and Saber (1998) and by El-Genk and Saber (1999); and the heat transfer coefficient by convection, $\mathrm{h}_{\infty}$, was calculated based on the values of experimental temperatures found in literature or experimented in UNESP - Guaratinguetá. 


\section{COMPUTATIONAL PROGRAM}

The solutions of the equation systems presented before was obtained from numerical procedure, based on the method of finite volumes. Within this context, the goal was to calculate the velocity fields $\left(\mathrm{w}^{*}, \mathrm{v}^{*}\right)$, pressure $\left(\mathrm{p}^{*}\right)$, temperature $(\mathrm{T})$ and density $\left(\rho^{*}\right)$, using the complete conservation equations.

The equations were solved in steady-state regime, being reasonable that next step was the transient implement for a possible comparison of results. But the unmotivation of the step was due to the results presented by Harley and Faghri (1994) which were of a pseudo transient, i.e., the solution was going to an answer in steady-state regime but the intermediated time did not correspond to experimental reality of Mingwei, Hongji and Qingming (1991), who had his thermosyphon starting from a freezing state. The proposed model in the present paper is not able to obtain results in a thermosyphon starting from a freezing state because under these conditions the vapor region is very scarce and the theory of the continuum is not valid. Furthermore, a possible comparison with the numerical results of Haley and Faghri (1994) would not be confident as his results weren't farther experimentally checked.

So, the motivation was in incorporating the reservatory of liquid in the complete solution of the conservation equations in steady-state regime. The incorporation of the reservatory of liquid was performed through the use of conservation equations in the resolution of the region of reservatory. Under these conditions, a problem was found. The correct value of the coefficient thermal conductivity inside the reservatory was vital to obtain confident results, but this value was unknown. Besides, the manipulation of this unknown took the program to obtain coherent or no-coherent results with the experimental.

The solution found was the one modeling the reservatory of liquid as if it was an unknown region, i.e., the thermal hydrodynamic phenomena occurred in the interior weren't studied, and its interference had to be represented in the boundary by the convective heat transfer coefficient. This is reasonable, because it's understood that this coefficient is parameter function as velocity, viscosity, density, specific heat, thermal conductivity, etc, attention was then transferred to the correct chosen of the convective heat transfer coefficient (El-Genk and Saber (1998) and El-Genk and Saber (1999)), that should be proper for using in a thermosyphon.

By knowing the convective heat transfer coefficient, assuming that the temperature of the reservatory is a arithmetical average of the temperatures in the section of interface between reservatory and vapor nucleus, considering the hydrostatic pressure of the reservatory and using Newton's cooling law, the temperature of the reservatory's wall is then a condition of boundary as shown in Region IV of Fig. 2 and the assembling with the rest of the pipe was performed.

The height of the reservatory of liquid was not assumed as variable parameter, so, the mass in the interior of the thermosyphon figured as a function of power transferred by the pipe, what is no physically realistic. By this supposition becomes acceptable when compared to the difficulties in obtaining a model for a thermosyphon, and furthermore, the implement of a liquid reservatory is a reasonable improving in theoretical modelings existent.

The method for the coupling of pressure-velocity used in this work is the SIMPLE (Semi-Implicit Method for Pressure-Linked Equations) based in Chorin's algorithm (1967) and (1971). The method SIMPLE, developed by Patankar and Spalding (Richtmyer and Morton (1967)), has the best pressure written as the adding of the best available pressure estimative, $p^{*}$, plus a correction $p$ that is calculated in a way to satisfy the equation of continuity.

The iterative method used in the solution of the linear system of equations was a mixture between the Gauss-Seidel method and method known as Thomas algorithm or TDMA (Tridiagonal Matrix Algorithm) (Patankar (1980), Richtmyer and Morton (1967), and Roache (1976)).

This way, a computational program was developed by FORTRAN language, with the goal of applying a method of finite volumes for the solution of equation system.

The program followed the next algorithm:

1- Initial.

2- Geometrical data input, axial point numbers and radial of the grid and properties of references.

3- Grid generation.

4- Calculus of the axial field.

5- Calculus of the radial field

6- Coupling pressure-velocity.

7- Calculus of the necessary parameters to the reservatory of liquid, such as: calculus of properties, calculus of average and hydrostatic pressure, checking the regime of operation (natural convection, combining convection or nucleate boiling).

8- Calculus of temperature field.

9- Calculus of liquid film thickness through Nusselts' classical theory.

10- Checking the convergence, if it doesn't satisfy the criterium return to item 3 .

11- The end.

As to the criteria of convergence adopted, the guarantee of convergence to no-linear systems is very hard to be obtained. Then, in order to control the convergence. Some factors of under-relation were imposed to the variables to avoid great corrections in a iteration and take to a divergence of solution.

The convergence must then be checked at each iteration and we should adopt a parameter that determines the end of the 
process of solution with the acquisition searched solution and this parameter is determinate by the chosen convergence criterium.

It is found in literature the use of several types of criteria, but in this paper the program was set free until the value of maximum medium residue, i.e., the maximum medium residue between the conservation equation find its minimum value, for each case. This procedure shows a real convergence of the program and besides, the residues were listed for each volume of control and the maximum exact value found in all the field will be shown through a variable named "sourax".

The choice of the number of points of the grid was performed to first find the case that would most cause instability in the computational program, among the cases to be investigated. Then, as shown in Fig. 3, a plot of the profile of outer wall temperature of the thermosyphon versus axial variation was done, for three numbers of different points of the grid

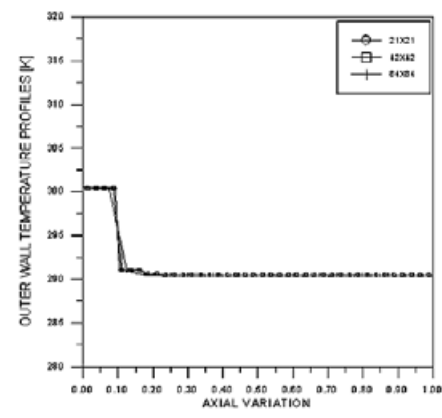

Figure 3. Test for the choice of the number points of the grid - case UFSC $50 \mathrm{~W}$.

In Fig. 3, it's noticed that there is an independence of results in relation to the number of points, as much axial as radial, through, it was observed that the time spent in CPU among cases $21 \times 21$ (radial $x$ axial) and $42 \times 42$ were not much different, but is was the opposite of case $84 \times 84$, that substantially increased the computational time. All the cases were done in a Pentium II $233 \mathrm{MHz}$ with $128 \mathrm{MB}$ of memory RAM microcomputer. In order to optimize with accuracy the results with the computational time, the grid $42 \times 42$ was chosen for all shown cases.

The view of the shown grid in Fig. 4, is the correspondent to the case that most caused instabilities in the computational program, it was the case of the pipe manufactured at Santa Catarina Federal University, specifically the one that transported 50W of thermal energy. Then impracticability of usage of a stretching factor is very clear. The three phases can be identified by the different sizes of the control volumes, and the region of the liquid-film, merely represented by a line. All the other cases have a similar grid.

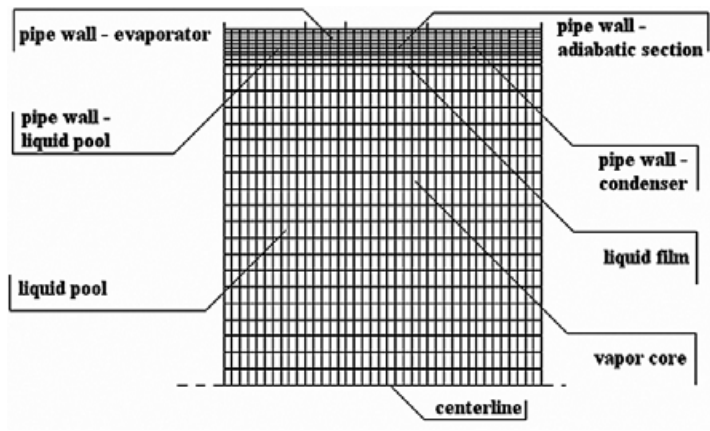

Figure 4. Computational grid - case UFSC $50 \mathrm{~W}$.

The technique of using a pseudo source to represent the changing phase when the energy conservation equation is written in Eq.(3) form was improved, and the experiment showed that this type of improvement causes serious instabilities to the computational program. During the interactive process, the pseudo source can assume a unreal value, taking the program to a divergence. Then solution found was the one guarantee the saturation temperature in all Region III of Fig. 2, uncoupling the solution between the vapor and liquid region. Both procedures were used and tested on Mingwei, Hongji and Qingming (1991) experimental case showing a good agreement, as shown later the results show that small differences occurred in relation to the field of velocities and eventually in the mass flowing rate, what does not mean errors in the calculus based on the shown results and confirming that the usage of conditions of saturating temperatures improve stability to the computational program this procedure was adopted for all the results to be shown later to Mingwei, Hongji and Qingming (1991) cases.

\section{EXPERIMENTAL DATA SPECIFIC COLLECT}

The tube filling scheme is shown in Fig. 5, while the tests system is constituted basically of:

a) A hot source made by a electric heating tubular resister, with a inner metal tube o promote homogenization of temperature in the oven.

b) A section of heat removing heat built from a PVC tube with internal chicanery tied to the thermosyphon to heat removing, circulation is performed, by gravity, in cool water, coming from a higher tank in steady level, through the space between two tubes.

c) A device that allows the gradient variation of the angle of the thermosyphon between $0^{\circ}$ and $90^{\circ}$, in relation to the horizontal position.

The instruments of this system is of 8 thermocouples along the thermosyphon for the measuring of the temperatures distribution, and it has thermocouples in the cooled water entrance and exit, to evaluation of transferred heat. 
Cooled water flowing were measured through a calibrate tank and chronometer and the potencies given by the oven were calculated through current and tension of resistance feeding.

Tests were performed with a thermosyphon built in copper, with $3 / 4$ "diameter, $0.60 \mathrm{~m}$ length, and $40 \%$ filling of the total thermosyphon volume. This way, through the oven power cooled fluid flowing adjustment, it was possible to simulate different working conditions, since low heat fluids up to values where the evaporation partial or total dryout occurred. The results used to check the computational program are shown in Fig. 6.

[1] Burette

[2] Vacuum meter

[3] Vacuum pump

[4] Valve

[5] Valve

[6] Capillary pipe

[7] Join

[8] Thermosyphon

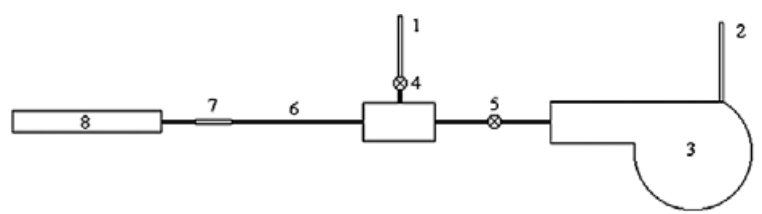

Figure 5. Scheme of the tube filling.

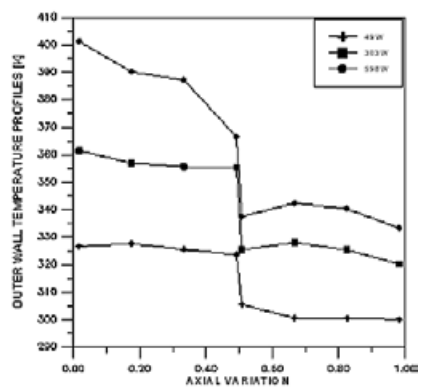

Figure 6. Experimental results.

\section{RESULTS DISCUSSIONS}

In order to promote a rigorous evaluations in the mathematical modeling used to represent the thermal hydrodynamics processes occurred in the interior of a cylindrical geometric thermosyphon on vertical operation, using water as working fluid, three cases were analyzed.

The first cases simulated a mild steel thermosyphon, experimental tested by Mingwei, Hongji and Qingming (1991), used in the parameter by Harley and Faghri (1994) mathematical modeling. A comparison of the model developed with experimental and theoretical data will be performed and commented later en.
The second case simulates a stainless steel thermosyphon, projected for application in bakery oven, using water as working fluid. The experimental tests were performed by Mantelli et al. (1999) in the Solar Energy Research Laboratory Federal University of Santa Catarina being tested three different values of heat transfer rate.

Finally, tests were performed at UNESP providing data to simulate a copper thermosyphon as described previously.

\section{First Case}

In Fig. 7, the behavior of the outer wall temperature, axially changing in the pipe is analyzed, with and without the use of pseudo source, showing a good agreement with the experimental results. The region that shows a more accented in relation to experimental results is between 0.32 and 0.61 approximately in relation to the length. Then maintenance of the height fixed of the liquid of the reservatory $(0.32)$ joined to the temperature degree that occurs to satisfy the model, promotes the appearing of the region with bigger difference. Physically, the appearing of higher temperatures in the region of the reservatory of liquid is coherent, due to the thermal dynamic processes attributed to this region, and that was experimentally verified by Mantelli et al. (1999). As to Harley and Faghri's (1994) results, the same does not occur, because in the respective model there aren't any reservatory of liquid and eventually the thermosyphon tends to equalize the temperature, this way not being capable to capture any degree of more accented temperature.

The first section presented in the numerical solution $(0$ to 0.32 ) represents the outer temperatures in the region of the reservatory of liquid, keeping in mind that this region was internally modeled through the heat transference coefficient, specifically developed for small reservatories where the numerical results end up to be influenced by a margin of errors of the expressions used.

Furthermore, a problem that can cause a increasing in the inaccuracy of numerical results is a false reading of thermocouples contained in this region of the evaporator, because due to the experimental coupling, these thermocouples are dependent to the thermal radiation influence, obtaining super estimated temperature values. A quantitative evaluation on the commented subject is necessary, but due to the lack of more details in literature, it's not possible.

Next section, comprehended between 0.32 and 0.45 is still the evaporator region, through the falling of temperature is accent because in this region there isn't a reservatory of liquid film. As the model does not foresee any increasing in the height of the reservatory of liquid during the functioning of the thermosyphon, this region becomes the function of the initial height of the reservatory. 


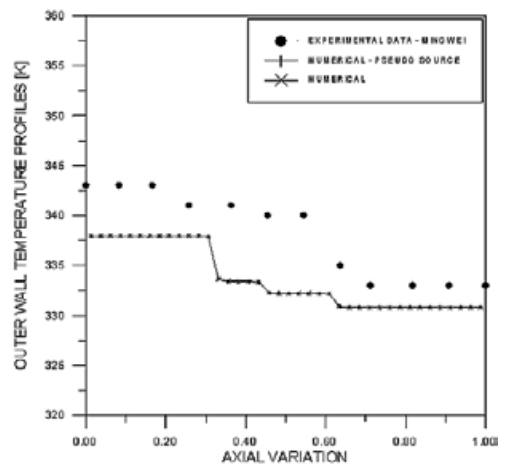

Figure 7. Outer temperature on the wall versus axial variation.

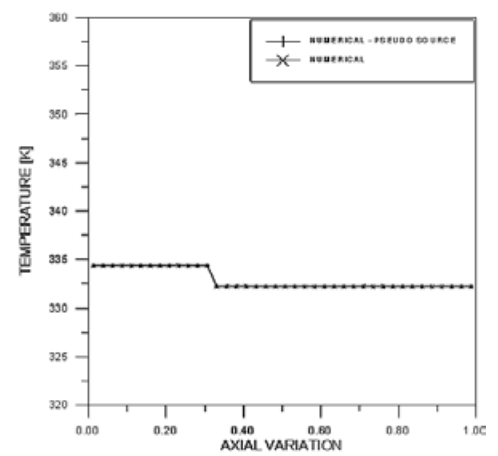

Figure 8. Internal temperature next to centerline versus axial variation.

The following section, comprehended between 0.45 to 0.64 represents the temperatures of adiabatic region, where the temperature level is slightly lower due to the outer boundary conditions adopted, of gradient of temperature null. Physically, this region is externally insulated, but there's a conduction of heat in the axial direction of the pipe emerging from the evaporator heating, what can cause an activity of fluid in this region, where theoretically the thickness of liquid film would be steady. This type of activity, added to a fixed maintenance of the height of he reservatory, can help in the deviation of the results. The last section, comprehend between 0.64 and 1.00, represents the temperature in the region of the condensator where the external boundary conditions used was a balancing of conduction and convection energy. In this region, the inner temperature joined to the value of the heat transference coefficient by external convection, end up to be important factors to determine the temperature field of the thermosyphon. It's noticed a good agreement between the numerical and experimental results.

In general, the presence of a pseudo source did not alter the numerical results, as can be observed by the superpositioning of results. The later performed tests cases from Santa Catarina Federal University and Engineering College from Guaratingue- tá - UNESP will not use this device anymore as its presence causes instabilities in the computational program convergence.

The variation of temperature next to the centerline, in relation to the adimensional length of the pipe was shown in Fig. 8, where it's clear the presence of a higher temperature in the reservatory of liquid in relation to the rest of the pipe. It's assumed that this increasing in temperature is connected to the physical phenomena occurred in this region, however in the model was assumed only the increasing of temperature related to hydrostatic pressure. The rest of the thermosyphon presents a uniform temperature, what is expected due to the only presence of a water vapor nucleus that is a region that presents a uniform pressure in most cases. Any variation was observed in relation to the pseudo source.

The behavior of adimensional axial velocity next to the centerline in relation to the adimensional length of the pipe was shown in Fig. 9. It's noticed that the graphic does not show the region of reservatory of liquid where it was not calculated the field of velocity and small variations occur due to the use of pseudo source. Then initial region (0.32) that represents the interface reservatory of vapor liquid nucleus, provides the value of injection velocity in the nucleus of vapor when the fluid changes phase. This velocity tends to increase partially because of the evaporation velocities coming from the liquid film, obtaining its maximum value in the exit section of the adiabatic region (0.64). In this region, the velocity field is little influenced by evaporation rates or condensation, that may really insulated. From this section up to the final edge, the opposite occurs, the velocities tend to diminish due to the influence of condensation rates, going up to zero when they reach the end of the thermosyphon.

The behavior of the thickness of liquid film in relation to the adimensional length of the pipe was shown in Fig. 10. No difference is noticed in relation

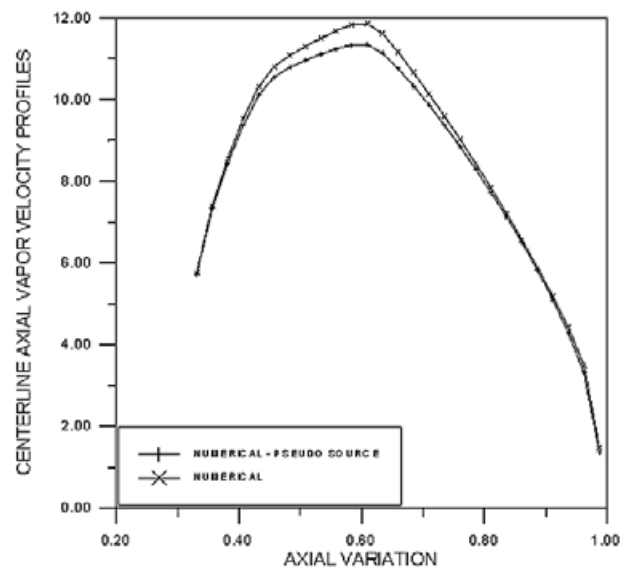

Figure 9. Axial velocity next to centerline versus axial variation. 
to the use of pseudo source. It's observed that in the reservatory of liquid was admitted a null thickness for a graphic representation purposes, what is coherent due to the evaporation rate present. In the adiabatic region, the program simulated the presence of a small rate of evaporation, because of this occurs a slightly inclination. From the 0.64 point, the thickness tends to decrease because in this region occurs condensation, and the limit is the condensator end cap when thickness is zero. It must be observed that thickness is influenced by gravitational action because the pipe is operation in vertical.

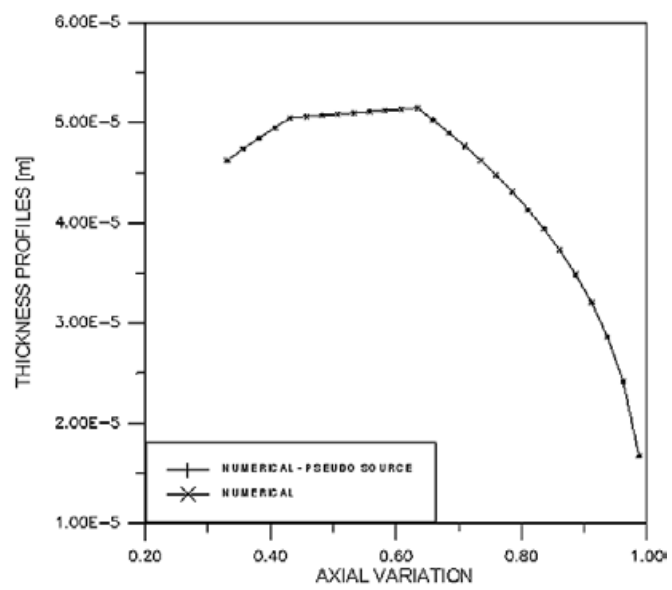

Figure 10. Thickness of liquid film versus axial variation.

The mass flowing rate variable with the pipe length was shown in Fig. 11, where a little difference is observed in relation to the use of pseudo source due to velocity field variation.

It's important to notice a good agreement between mass flowing rate and mass liquid, as much when used a pseudo source as in the case when not used a pseudo source. This result is expected in all sections of the pipe when it's operating in steadystate regime. An increase in the mass flowing rate at the top of condensator up to the adiabatic region is expected because as the liquid film returns by gravitational action to the reservatory of liquid, more liquid mass accumulates. In the adiabatic section the case in which it was used a pseudo source, shows a sensitive increase in the flowing rate in relation to the case where it was not used pseudo source, as it gets closer to the evaporator. The explanation can be justified in the user of pseudo source only in the condensator region, keeping this way the boundary saturation condition for the adiabatic region and condenser, taking the program to adjust similar values was not used.

The axial adimensional velocity component in certain sections, radialy varying was shown in Fig. 12 where a little difference in velocity profile is present in the result when the use or not use of pseudo source in profile for the adiabatic section. As expected, in adiabatic section the bigger profiles of velocity are present, satisfying the mass conservation equation, while flowing effects in counter current of vapor can be observed in the final end section of condensator, as it gets closer to the liquid film.

A more adequate visualization of velocity profiles in the liquid film can be observed in Fig .13 where the opposite film nature is clear. The adequate studies of velocity profiles in the vapor nucleus and in liquid films is important to a better evaluation of operation limits of the thermosyphon. One of the limits, known as flooding limits occurs when the velocity profile of vapor is accented, increasing the pulling force that acts in the liquid film, making the liquid mass flowing rate tend to zero. This causes the evaporation dry out. Faghri, Chen and Morgan (1989) determined that this fenomenum is of periodic nature, with oscillation period of dry out dependent of the magnitude of the heat provided to the thermosyphon.

To complete the analysis, the graphics of residue are represented in Fig. 14 and Fig. 15, where the peak in both graphics does not correspond to a natural oscillation of the program, but to a correction of the reference pressure that was necessary to satisfy the requirements of the heat transfer of the pipe.

In both cases, the maximum residues (soraux) found in the whole field was relatively low when compared to the initial medium maximum residue.

\section{SECOND CASE}

Comparative graphics with experimental tests are presented, intending to interpret he behavior of the thermosyphon outer temperatures in certain operation conditions. Furthermore this developed computational program goes through more evaluation in which two additional situations are tested and compared to experimental results. At first situation, the calculus of the reservatory of liquid temperature is submitted to a parameter of entrance of program, while in the second situation, besides the temperature

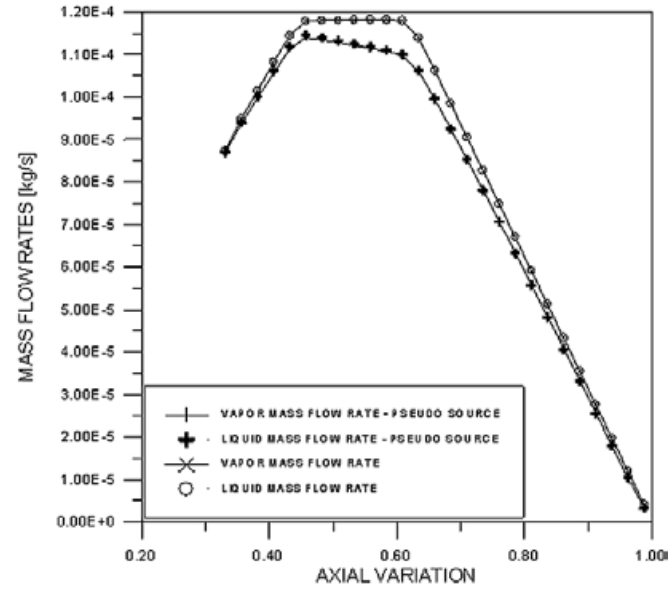

Figure 11. Mass flow rates versus axial variation. 


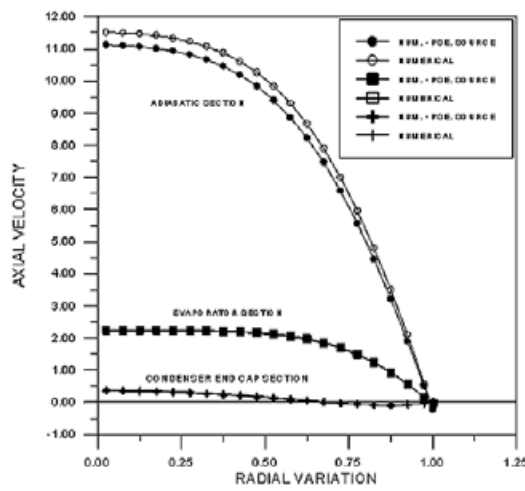

Figure 12. Axial velocities versus radial variation.

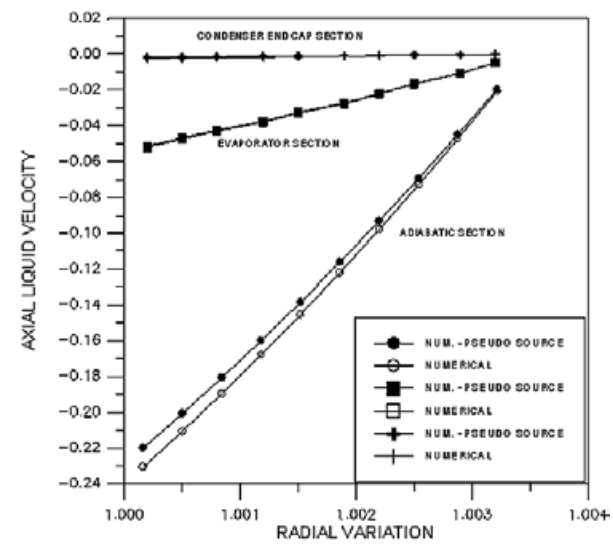

Figure 13. Axial velocities of liquid film versus radial variation.

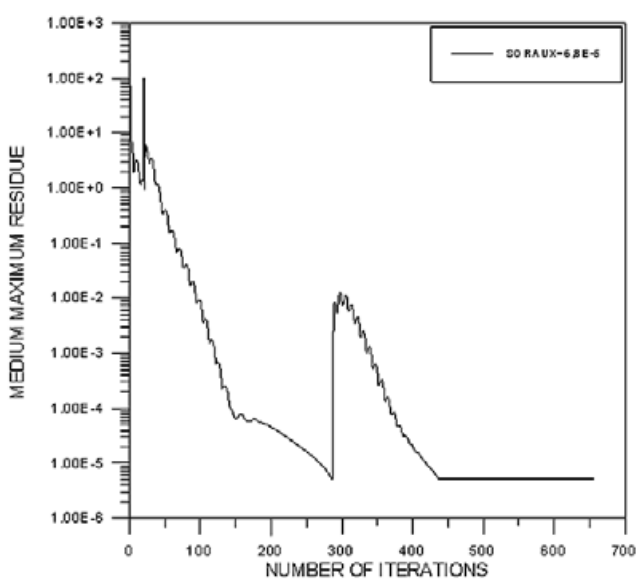

Figure 14. Medium maximum residue versus number of iterations using pseudo source.

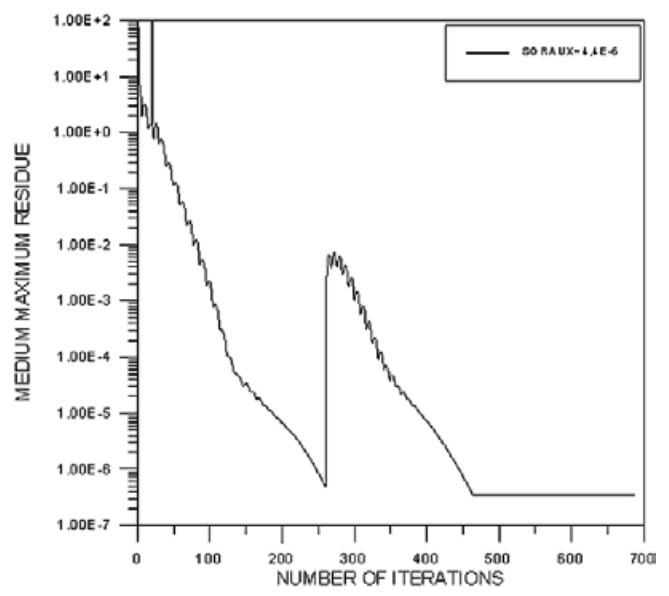

Figure 15. Medium maximum residue versus number of iterations without pseudo source.

the heat transfer coefficient by convection of the reservatory of liquid becomes a parameter of entrance of the computational program. It's important to point out that as much the temperature as the heat transfer coefficient by convection of the reservatory of liquid were obtained from the experimental data, so enabling its use as parameters of entrance in the computational program. In Fig. 16, the behavior of the thermosyphon outer temperatures in relation to its adimensional length was evaluated with experimental data of a pipe transferring $50 \mathrm{~W}$. The numerical test did not present a good agreement with the experimental results showing that the model used or the reservatory of liquid was not able to simulate the physical phenomena occurred in this region. In opposite, a significant improvement was obtained when using the temperature of the reservatory (TP) as a parameter of entrance of the program, and also in the case of temperature joined to the heat transfer coefficient by convection of the reservatory (TP and HPIS). This shows the consistence of the assembling of the computational program, and furthermore part of the difference of temperature presented in the region of the reservatory of liquid, among the experimental and theoretical results must be associated to the assembling procedures of tests systems, i.e., the thermocouples can be determining temperatures a little higher to reality, due to the effects of radiation from the oven. The three theoretical cases presented did not use the pseudo source, for already commented reasons. The cases of pipes transferring $300 \mathrm{~W}$ and $600 \mathrm{~W}$ have a similar analysis to the already discussed cases of $50 \mathrm{~W}$.

Only some commentaries are interesting, as the better agreement with the experimental results shown in Fig. 17, when it's used a temperature value of reservatory (TP) and film coefficient (HPIS) known in relation to others, while in Fig. 18 a superheating was detected. It's important to point out that there's a great chance for pipes with small filling 
charges presented a functioning standard, known as nuclead boiling in the region of the reservatory of liquid. This type of functioning causes an expansion at the height of the reservatory of liquid due to the presence of bubbles in its interior. As the model does not foresee this, it ended up to occur a region of bigger inconsistency in the results, always neat to the adiabatic section.

\section{THIRD CASE}

Three different power cases transferred by the thermosyphon were examined and compared to the numerical procedures, besides temperature tests performed and reservatory heat transfer coefficient assumed as known - calculated through a estimative among the outer temperatures of evaporation and condensator - and used input parameters.

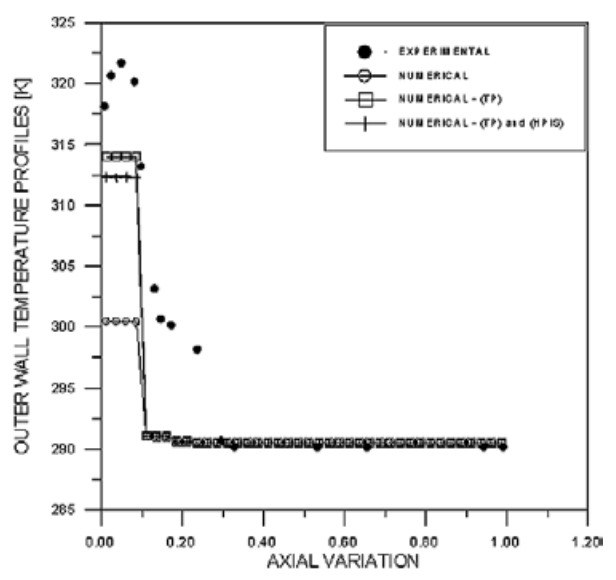

Figure 16. Outer wall temperature versus axial variation.

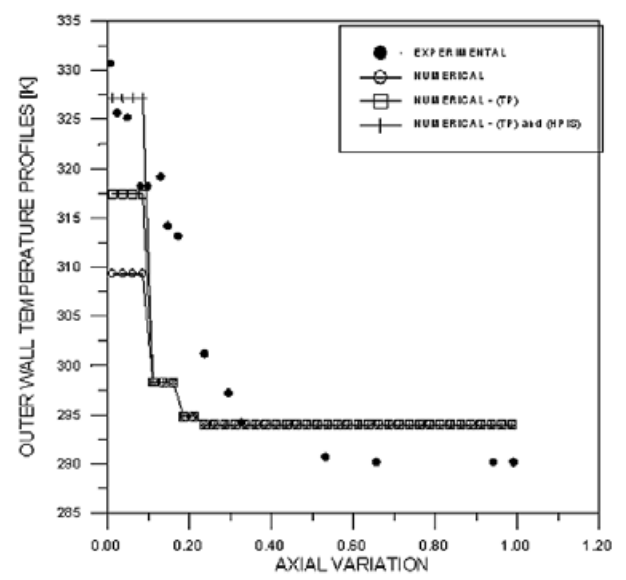

Figure 17. Outer wall temperature versus axial variation.

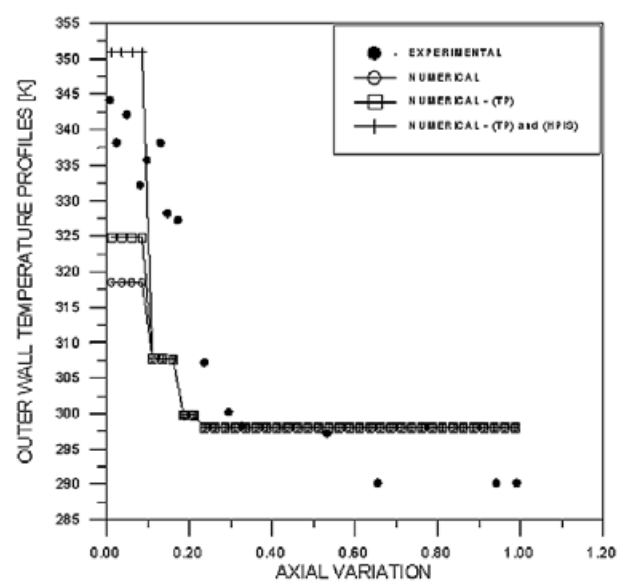

Figure 18. Outer wall temperature versus axial variation.

This last evaluation of the computational program was performed with the thermosyphon manufactured at UNESP. Three transferred potencies were checked and in general they shoed behavior similar to those already discussed; Fig. 19, Fig. 20, Fig. 21 show the behavior of the pipe outer temperature in relation to its adimensional length and shoed better agreement to the experimental results for the cases where the temperature of reservatory (TP) and the coefficient of film (HPIS) are input parameters of the program. As mentioned before, better results were not obtained probably because of the fixed height of the reservatory of liquid, what causes a more intense deviation of reality in the region between evaporator and condenser.

\section{FINAL CONCLUSIONS}

Intending to obtain a computational program able to approximate theory and pratic as much as possible, a bidimensional modeling using the conservation equations was implemented and tested with data from literature and data obtained experimentally, through a system of tests built for this purpose. Although during the development of the

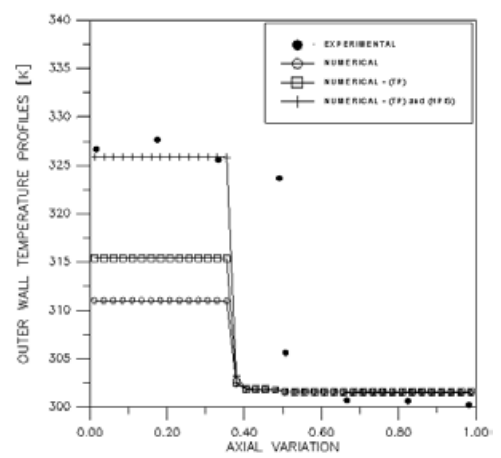

Figure 19. Outer wall temperature versus axial variation. 


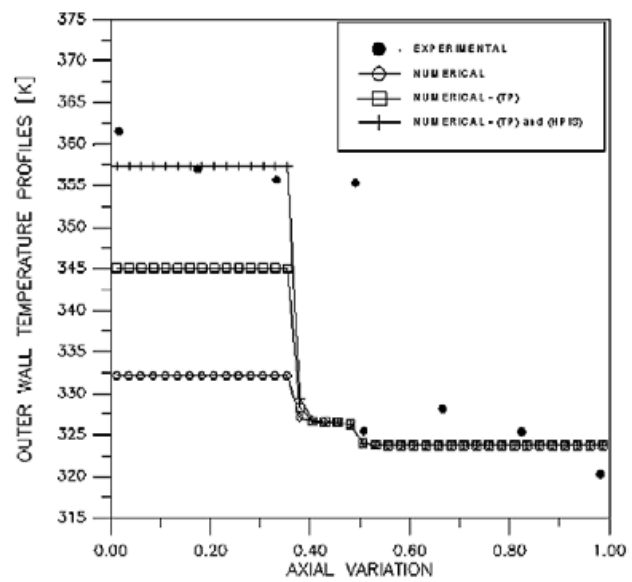

Figure 20. Outer wall temperature versus axial variation.

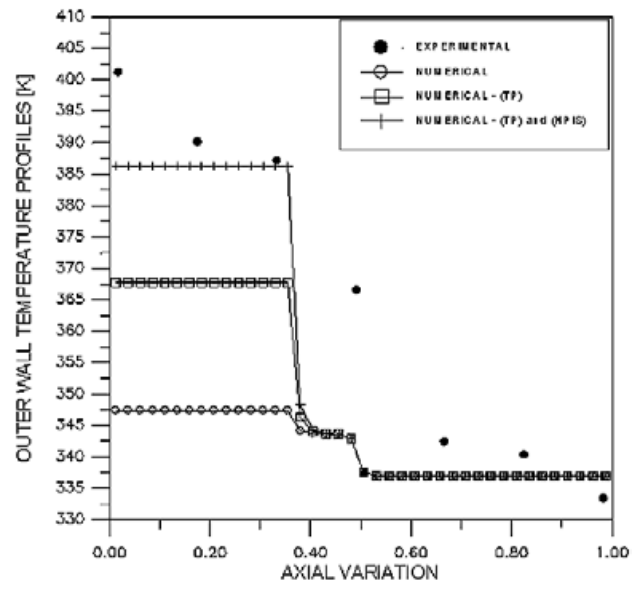

Figure 21. Outer wall temperature versus axial variation.

work the transient terms in mathematical modeling were conserved, the program intended to obtain results for the steady-state regime, however, assuming the presence of a reservatory of liquid.

A very important consideration is that the total mass contained in the pipe interior is a fixed parameter. However, when keeping the height of fixed reservatory, the pipe can present a fluid mass increasing when it's increased the input heat flow. This is necessary to satisfy the new requirements of heat transfer and the new mass amount occurs with the increasing of thickness of liquid film. But even being physically incoherent, the mass amount is reasonably small.

Only the conservation equations weren't enough to capture the physical effects in the interior of a small reservatory under the influence of a heat flow. The need of an expression able to calculate a thermal conductivity adequate to the phenomenum is essential. As this is not reality yet, the way found to solve this problem was the one of modeling the reservatory assuming only its boundary effects. This way, all the physical representation was transference rate between the fluid and the reservatory internal wall. A proper knowledge of the fluid temperature inside the reservatory is also necessary. Some disagreements on the results were obtained in consequence of a week foresee of the theoretical modeling used in literature for the reservatory of liquid, what did not unable the use of the program. A way found to solve this problem was the using of real values experimentally obtained, as much in temperatures as in heat transference coefficient of the reservatory of liquid, as input parameters of the computational program. This way, the bigger inaccuracy was the expansion of the height of reservatory of liquid with the pipe in operating. It's valid to remember that this modeling is strongly different from the one presented by Harley and Faghri (1994) because the conservation equations were totally solved in the interior of the liquid film and the effects of the presence of a reservatory are included.

\section{ACKNOWLEDGEMENTS}

The authors thank to CAPES (Brazil) for the financed support.

\section{REFERENCES}

Chorin, A. J., 1967, A Numerical Method for Solving Incompressible Viscous Flow Problems, Journal of Computational Physics, Vol. 2, pp. 12-26.

Chorin, A. J., 1971, Numerical Solution of the NavierStokes Equations, Math. of Computation, Vol. 22, pp. 745-762.

El-Genk, M. S., and Saber, H. H., 1999, Determination of operation envelopes for closed, two-phase thermosyphons, International Journal of Heat and Mass Transfer, Vol. 42, pp. 889-903.

El-Genk, M.S., and Saber, H. H., 1998, Heat Transfer correlations for small, uniformly heated liquid pools, Int. J. Heat Mass Transfer, Vol. 41, No. 2, pp. 261-274.

Faghri, A., Chen, M. M., and Morgan, M., 1989, Heat Transfer Characteristics in Two-Phase Closed Conventional and Concentric Annular Thermosyphons, ASME Journal of Heat Transfer, Vol. 111, pp. 611-618.

Ganic, E. N., Hartnett, J. P., and Rohsenow, W. M., 1985, Basic Concepts of Heat Transfer: Handbook of Heat Transfer Fundamentals, New York: McGraw-Hill.

Harley, C., and Faghri, A., 1994, Complete Transient TwoDimensional Analysis of Two-Phase Closed Thermosyphons Including the Falling Condensate Film, Journal Heat TransferTransactions of the ASME, Vol. 116, pp. 418-426.

Irvine Jr., T. F., and Liley, P. E., 1984, Steam and Gas Tables with computer equations, Academic Press.

Mantelli, M. B. H., Carvalho, R. D. M., Colle, S., and Moraes, D. U. C., 1999, Study of Closed two-phase thermosyphon for bakery oven applications, Proceedings of 
$33^{\text {rd }}$ National Heat Transfer Conference, Albuquerque, New Mexico.

Mingwei, T, Li, C., Hongji, Z., and Qingming, T., 1991, Start-Up Behavior of the Frozen Two-Phase Closed SteelWater Thermosyphons, Proc. $8^{\text {th }}$ Heat Pipe Conference, B-P2.

Patankar, S. V., 1980, Numerical Heat Transfer and Fluid Flow, Washington, D.C: Hemisphere.

Richtmyer, R. D., and Morton, K. W., 1967, Difference Methods for Initial-Value Problems, New York: John Wiley $\&$ Sons.

Roache, P. J., 1976, Computational Fluid Dynamics, Hermosa.

Received: September 06, 2006

Revised: October 06, 2006

Accepted: November 06, 2006 